Internist 2014 · 55:1112-1112 • DOI 10.1007/s00108-014-3576-9

Online publiziert: 5. September 2014 • @ Springer-Verlag Berlin Heidelberg 2014

G. Klose ${ }^{1} \cdot$ F.U. Beil' $\cdot$ H. Dieplinger ${ }^{3} \cdot$ A. von Eckardstein ${ }^{4} \cdot$ B. Föger ${ }^{5}$.

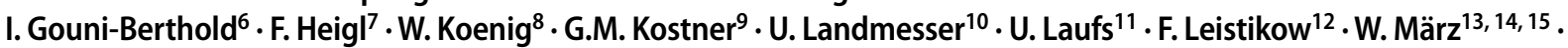
M. Merkel ${ }^{16}$. D. Müller-Wieland ${ }^{17}$. G. Noll ${ }^{10}$. K.G. Parhofer ${ }^{18}$. B. Paulweber ${ }^{19}$. W. Riesen ${ }^{20}$. J.R. Schaefer ${ }^{21}$. E. SteinhagenThiessen $^{22,23} \cdot$ A. Steinmetz ${ }^{24} \cdot$ H. Toplak ${ }^{25} \cdot$ C. Wanner ${ }^{26} \cdot$ E. Windler $^{27}$

\title{
Erratum zu: Neue AHA- und ACC-Leitlinie zur Risikoreduktion von Herz-Kreislauf-Erkrankungen durch Cholesterinsenkung. Stellungnahme der D•A-CH-Gesellschaft Prävention von Herz- Kreislauf-Erkrankungen e. V., der Österreichischen Atherosklerose Gesellschaft und der Arbeitsgruppe Lipide und Atherosklerose (AGLA) der Schweizer Gesellschaft für Kardiologie
}

Versehentlich wurde ein Autor im Beitrag nicht aufgeführt und die Affiliationen der Autoren z. T. nicht korrekt wiedergegeben. Wir bitten, zukünftig die korrekte Nennung zu beachten und die Fehler zu entschuldigen.

\section{Korrespondenzadresse}

\section{Prof. Dr. E. Windler}

Präventive Medizin, Klinik und Poliklinik für Allgemeine und Invasive Kardiologie, Universitäres Herzzentrum, Universitätsklinikum Hamburg-Eppendorf

Martinistr. 52, Haus N36, 20246 Hamburg windler@uke.uni-hamburg.de
Die Online-Version des Originalartikels können Sie unter http://dx.doi.org/10.1007/s00108-0143492-z finden.

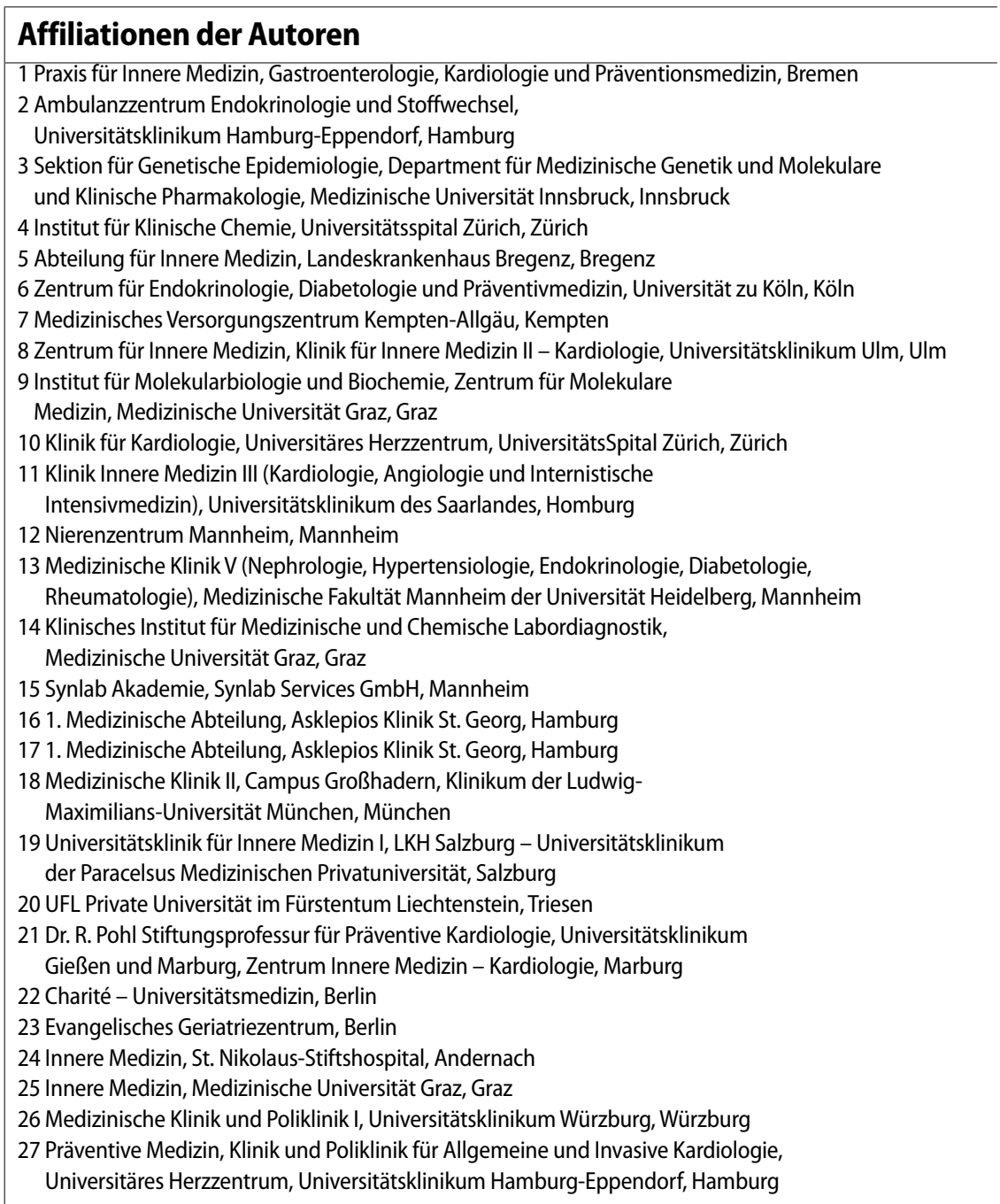

1 Praxis für Innere Medizin, Gastroenterologie, Kardiologie und Präventionsmedizin, Bremen

2 Ambulanzzentrum Endokrinologie und Stoffwechsel,

Universitätsklinikum Hamburg-Eppendorf, Hamburg

3 Sektion für Genetische Epidemiologie, Department für Medizinische Genetik und Molekulare

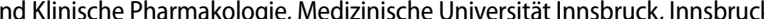

5 Abteilung für Innere Medizin, Landeskrankenhaus Bregenz, Bregenz

6 Zentrum für Endokrinologie, Diabetologie und Präventivmedizin, Universität zu Köln, Köln

7 Medizinisches Versorgungszentrum Kempten-Allgäu, Kempten

8Zentrum für Innere Medizin, Klinik für Innere Medizin II - Kardiologie, Universitätsklinikum Ulm, Ulm

Medizin, Medizinische Universität Graz, Graz

10 Klinik für Kardiologie, Universitäres Herzzentrum, UniversitätsSpital Zürich, Zürich

Medizinische Universität Graz, Graz

15 Synlab Akademie, Synlab Services GmbH, Mannheim

16 1. Medizinische Abteilung, Asklepios Klinik St. Georg, Hamburg

71. Medizinische Abteilung, Asklepios Klinik St. Georg, Hamburg

Maximilians-Universität München, München

19 Universitätsklinik für Innere Medizin I, LKH Salzburg - Universitätsklinikum

Gießen und Marburg, Zentrum Innere Medizin - Kardiologie, Marburg

Charité - Universitätsmedizin, Berlin

26 Medizinische Klinik und Poliklinik I, Universitätsklinikum Würzburg, Würzburg Universitäres Herzzentrum, Universitätsklinikum Hamburg-Eppendorf, Hamburg 